\title{
Retrosigmoid Approach for Resection of Cerebellopontine Angle Meningioma
}

\author{
Hussam Abou-Al-Shaar ${ }^{1} \quad$ Yair M. Gozal ${ }^{1} \quad$ Gmaan Alzhrani $^{1} \quad$ William T. Couldwell ${ }^{1}$ \\ ${ }^{1}$ Department of Neurosurgery, Clinical Neurosciences Center, \\ University of Utah, Salt Lake City, Utah, United States \\ J Neurol Surg B 2018;79(suppl S5):S407-S408.

\begin{abstract}
Address for correspondence William T. Couldwell, MD, PhD, Department of Neurosurgery, Clinical Neurosciences Center, University of Utah, 175 North Medical Drive East, Salt Lake City,
\end{abstract} \\ UT 84132, United States (e-mail: neuropub@hsc.utah.edu).
}

\begin{abstract}
Keywords

- meningioma

- retrosigmoid approach

- cerebellopontine angle

- craniotomy

This video depicts the case of an 81-year-old man who presented with a 3-month history of left-sided facial numbness and gait imbalance. On examination, he had gait ataxia and decreased left facial sensation in the V2 and V3 distribution. Magnetic resonance imaging (MRI) revealed a large homogenously enhancing lesion arising from the inferior surface of the tentorium toward the left cerebellopontine angle causing significant brainstem compression (-Fig. 1A and 1B). The differential diagnoses for this lesion included meningioma and trigeminal nerve schwannoma. Given the patient's symptoms, the size of the lesion, and the severity of brainstem compression, surgical resection was recommended. The patient underwent a left retrosigmoid craniotomy for resection of the mass (-Fig. 2). The patient tolerated the procedure well with no new postoperative neurological deficit. Histopathological examination of the lesion revealed a World Health Organization (WHO) grade I meningioma. Postoperative MRI was consistent with gross total resection of the tumor ( - Fig. 1C and 1D). The patient was discharged home on postoperative day 4. At his last follow-up appointment, 1 month after surgery, the patient reported complete resolution of his imbalance and left-sided facial numbness. The patient gave consent for publication.

The link to the video can be found at: https://youtu.be/xoHmhv7bme4.
\end{abstract}

Conflict of Interest

None.

Acknowledgments

We thank Vance Mortimer for assistance with the video and Kristin Kraus for editorial assistance.

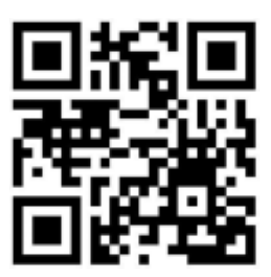

received

May 22, 2018

accepted

August 12, 2018

published online

September 25, 2018 www.thieme.com/skullbasevideos

www.thieme.com/jnlsbvideos

(c) 2018 Georg Thieme Verlag KG
Stuttgart · New York

License terms

10.1055/s-0038-1669975.

ISSN 2193-6331. (c) (1) $\ominus$ (\$) 

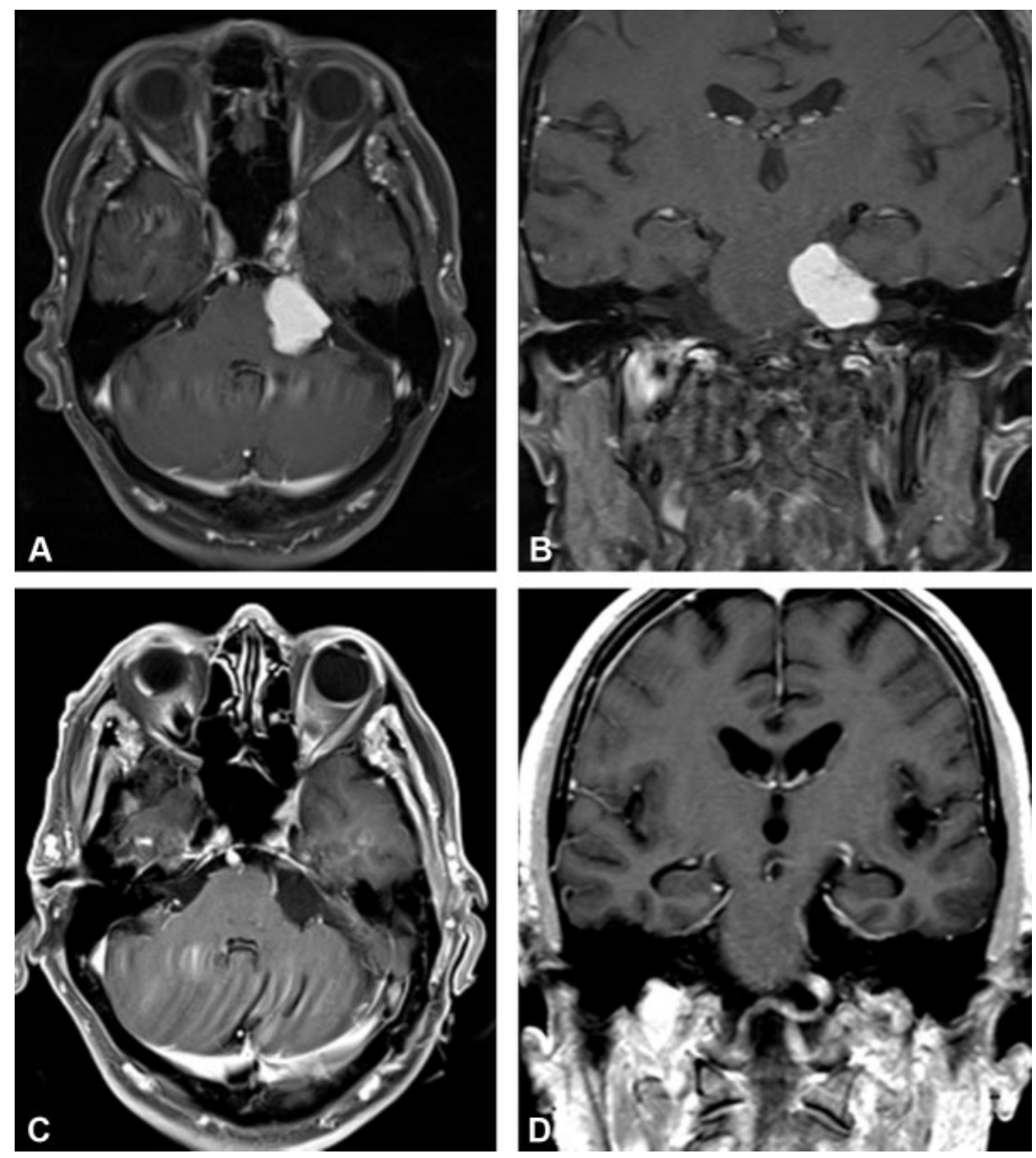

Fig. 1 (A) Preoperative axial and (B) coronal magnetic resonance images of a cerebellopontine angle meningioma, causing significant brainstem compression. (C, D) Postoperative magnetic resonance images (C, axial; D, coronal), showing gross-total resection.

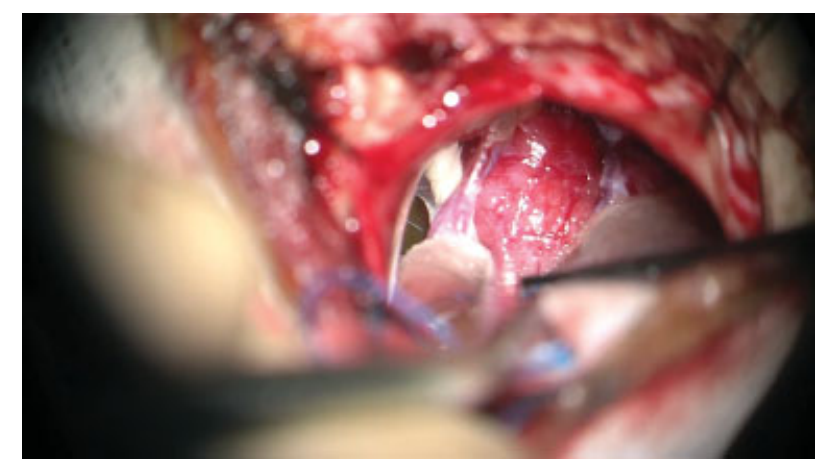

Fig. 2 Intraoperative image showing left retrosigmoid craniotomy for resection of the mass. 